\title{
PERITONEAL DIALYSIS RELATED PERITONITIS AS A RISK FACTOR FOR CARDIOVASCULAR EVENTS
}

Author details:

Kirsten S. Hepburn ${ }^{1}$, Renal Advanced Trainee, Wollongong Hospital.

Kelly Lambert ${ }^{2}$, Academic Program director Nutrition and Dietetics, School of Medicine, Faculty of Science, Medicine and Health, University of Wollongong. Research Fellow, Illawarra Shoalhaven Local Health District, Department of Nutrition and Dietetics, Wollongong Hospital. Research Fellow, Illawarra Health and Medical Research Institute University of Wollongong.

Judy Mullan², Director of Centre for Health Research Illawarra Shoalhaven Population (CHRISP).

Brendan McAlister ${ }^{2,3}$, Integration development coordinator, Illawarra Shoalhaven local health district.

Maureen Lonergan ${ }^{1,4}$, Senior Staff Specialist Nephrology Illawarra Shoalhaven Local Health District

Hicham I. Cheikh Hassan ${ }^{1,4}$, Staff Specialist Nephrology Illawarra Shoalhaven Local Health District

Author Affiliations:

${ }^{1}$ Department of Renal Medicine, Wollongong Hospital NSW, Wollongong, NSW, Australia

${ }^{2}$ Centre for Health Research Illawarra Shoalhaven Population (CHRISP), University of Wollongong, Wollongong, NSW, Australia 
3 Illawarra Shoalhaven Local Health District, NSW, Australia.

${ }^{4}$ University of Wollongong, Wollongong, NSW, Australia

All Authors contributed significantly to the manuscript and are in agreement with the content. K.H analysed the data and wrote the manuscript in close consultation with H.CH. K.L, J.M and B.M facilitated data linkage and provided data for analysis and reviewed the manuscript providing feedback. M.L. reviewed the manuscript and provided feedback.

\section{ACKNOWLEDGEMENTS}

The authors acknowledge the CHRISP research partnership established between the Illawarra Shoalhaven Local Health District (ISLHD) and the University of Wollongong, and ISLHD for funding support and as the source of data used in this study. We acknowledge the Australian and New Zealand Dialysis and Transplant registry (ANZDATA) for assisting with the data acquisition, as well as all of the contributors who voluntarily provide information to the registry.

\section{Corresponding author:}

Dr Hicham I. Cheikh Hassan, Department of Renal Medicine, 3 Dudley Street, Wollongong NSW 2500, Australia Hichamibrahim.chiekhhassan@health.nsw.gov.au. 0242225443. 


\section{INTRODUCTION}

Chronic kidney disease (CKD) is becoming increasingly common, with an estimated incidence of $10 \%$ in Australian adults, and more than $40 \%$ in people aged 75 and over $^{1}$. Progression of CKD leads to end stage kidney disease (ESKD) and ultimately the need for renal replacement therapy (RRT), with an incidence of 124 per million population in Australia in $2017^{2}$. Peritoneal dialysis (PD) is one modality of RRT, accounting for around $19 \%$ of all Australian dialysis patients ${ }^{3}$. Compared to haemodialysis, PD is often the preferred modality due to preservation of residual renal function, which may mitigate cardiovascular risk; improved early survival as well as convenience and flexibility for patients such as the ability to travel ${ }^{4}$. The main complication of PD is peritonitis, with an incidence of 31 per 100 patient years in Australian PD patients ${ }^{3}$.

CKD is associated with up to a 20 -fold increase in risk of cardiovascular death compared to patients without $C K D^{5}$. In Australian PD patient's, cardiovascular disease (CVD) is the leading cause of mortality accounting for up to $35 \%$ of deaths ${ }^{6}$. While there is a high prevalence of cardiovascular risk factors in patients with CKD, this does not completely explain the excessive cardiovascular risk experienced by this population ${ }^{5,7-8}$. This may be attributed to the important biochemical and physiological changes associated with CKD including; abnormal calcium and phosphate metabolism, malnutrition, inflammation, enhanced coagulability, anaemia, uraemic toxicity, fluid overload, vascular calcification and endothelial dysfunction ${ }^{5,7,9-10}$. In the PD 
population, high glucose concentrations in PD fluid may contribute to dyslipidaemia and insulin resistance $^{5}$ and exposure to bio-incompatible PD fluid and glucose degradation products (GDPs) may contribute to a state of chronic inflammation, all of which increase cardiovascular risk ${ }^{7,10}$. Furthermore, PD fluid and GDPs lead to the accumulation of reactive oxygen species which are thought to play a key role in endothelial damage and atherosclerosis ${ }^{11}$.

It is well recognised that patients with CKD are at increased risk of infection compared to those without $\mathrm{CKD}^{8,12-13}$, with more severe infective episodes ${ }^{12}$ and an increased associated mortality ${ }^{12,14}$. This may be due to impaired immunity, reduced vaccination responsiveness, significant comorbidities, and frequent exposure to healthcare facilities ${ }^{13}$. An association between infection and CVD has been demonstrated in the general (non-CKD) population ${ }^{15-16}$ and in nondialysis CKD populations ${ }^{8}$. In the haemodialysis population, bacteraemia or septicaemia is associated with a $78 \%$ increase in risk of myocardial infarction ${ }^{17}$, while infection related hospitalisations in older dialysis patients increase the risk of cardiovascular events in the following 30 days by $25 \%{ }^{18}$. Moreover, PD patients are at higher risk of death from CVD after the first year of treatment compared to haemodialysis patients and this is presumed, in part, to be due to increased infectious episodes over time ${ }^{5}$.

Peritonitis has been associated with increased risk of mortality, with one Australian study demonstrating a $19 \%$ mortality in PD patients within 30 days of a peritonitis episode ${ }^{19}$. It is hypothesized that systemic inflammation resulting from peritonitis may predispose patients to cardiovascular events ${ }^{12}$, however there is a paucity of research examining this association in the PD population. While a recent study established an association between peritonitis and late cardiovascular mortality in PD patients ${ }^{7}$, no studies have examined the association between peritonitis and all cardiovascular events in this population. We aimed to examine if peritonitis is a 
risk factor for all cardiovascular events in PD patients. We hypothesised that an episode of peritonitis increases the risk of future cardiovascular events in patients undertaking PD. 


\section{MATERIALS AND METHODS}

We conducted a retrospective cohort study of PD patients from one health district in New South Wales, Australia. We included adults, aged 18 years and over, who commenced PD from 2001 to 2015. Patients were excluded if they were on PD for $<3$ months, previously had a renal transplant, or underwent haemodialysis previously for $\geq 6$ months, had recovered their renal function, or had commenced PD in another area health service (Figure 1). At the study design phase we calculated the sample size required. For a power of 0.8 at an alpha level of 0.05 we anticipated that the Hazard Ratio for cardiovascular events from infection to be 2.0 (based on studies for chronic kidney disease showing a HR of 1.8- 3.0) ${ }^{8}$. We also anticipated for the exposure (peritonitis) rate based on the ANZDATA to be $60 \%{ }^{3}$ and the event rate (cardiovascular events) to be $50 \%$. Assuming a drop-out rate of $15 \%$ the sample size calculated was 225 . This study was approved by the University of Wollongong and Illawarra Shoalhaven Local Health District Health and Medical Human Research Ethics Committee (2017/266).

Baseline sociodemographic, laboratory and clinical data was obtained from the Australian and New Zealand Dialysis and Transplant (ANZDATA) registry. Data included gender, age, ethnicity, primary renal disease, height and weight (used to calculate Body Mass Index), comorbidities and smoking status at commencement of RRT. Comorbidities included chronic lung disease, coronary artery disease, peripheral vascular disease, cerebrovascular disease, diabetes and any diagnosis of malignancy. Baseline laboratory results included creatinine (mmol/L), uncorrected calcium (mmol/L), phosphate $(\mathrm{mmol} / \mathrm{L})$ and haemoglobin $(\mathrm{g} / \mathrm{L})$. Primary renal disease causing ESKD was classified as diabetes, hypertension, glomerulonephritis, cystic, or other renal disease. Peritonitis episode data, relocation data, transition between dialysis types, and cause of 
death were also obtained from the ANZDATA registry which captures this information in real time. As part of this study, we included all peritonitis events prior to cardiovascular events.

The primary outcome was any cardiovascular event, defined as a composite of hospitalization for cardiovascular ischaemia or infarction, unstable angina, congestive cardiac failure, ischaemic stroke or transient ischaemic attack; and cardiovascular death. Cardiovascular death was defined as death from cardiac arrest of unknown cause, death from pulmonary oedema, death from myocardial ischemia or infarction, and death from cerebrovascular event. Cardiovascular event data was obtained from the ANZDATA registry and from International Statistical Classification of Disease and Related Health Problems $10^{\text {th }}$ revision (ICD-10) coding and procedure block numbers using the Australian Classification of Health Interventions (ACHI) codes. Data linkage was facilitated by the Centre for Health Research Illawarra Shoalhaven Population (CHRISP). The ICD-10 codes G45.9 (Transient cerebral ischaemic attack, unspecified), I20.0 (Unstable angina), I21 (Acute myocardial infarction), I22 (Subsequent myocardial infarction), 146 (Cardiac arrest), 150 (Heart failure) and 163.9 (Cerebral infarction, unspecified) were used to identify inpatient cardiovascular events. ACHI codes 667 (Cardiac Catheterisation), 668 (Coronary angiography), 670 (Transluminal coronary angioplasty), 671 (Transluminal coronary angioplasty with stenting), and 672-679 (Coronary artery bypass) were also used. Procedure codes were used to identify admissions, however an intervention alone in the absence of a cardiovascular event as defined above was not included as an outcome.

Patients were censored after their first cardiovascular event, or at time of death from noncardiac cause, transfer to another NSW health district for more than 30 days or at the end of the study period (31 $1^{\text {st }}$ December 2015). Categorical data, expressed using frequency (\%), was examined by Chi-Square tests. Continuous data was expressed as medians with interquartile 
ranges (IQR) $\left[25^{\text {th }}-75^{\text {th }}\right.$ percentile] or mean with standard deviation (SD) and compared by Mann Whitney or Independent samples t-test as appropriate. Cox regression models were used to estimate crude hazard ratios (HR) with $95 \%$ confidence intervals $(95 \% \mathrm{Cl})$ for cardiovascular events. Time-dependent covariate analysis using Cox proportional models were used to determine adjusted HR with $95 \% \mathrm{Cl}$. The models accounted for a time dependent covariate from date of commencing PD as the start of follow up time until the first peritonitis episode. Covariate selection for the final model was based on univariate significance, plausible confounders, established risk factors and a backwards stepwise model. Statistical analysis was performed using SPSS version 23.0. 


\section{RESULTS}

We identified 262 patients who commenced PD between 2001 and2015, 51 patients were excluded (Figure 1). We included a total of 211 patients in the analysis with a median follow up time of 3.5 years (IQR $1.4-6.0$ years). The study population had a median age of 66 years (IQR 54.5 - 74.5 years), was predominantly male (64.0\%), Caucasian (94.3\%), former smokers (46.9\%) and overweight with a mean body mass index (BMI) of $28 \mathrm{~kg} / \mathrm{m}^{2}\left(\mathrm{SD} 5.1 \mathrm{~kg} / \mathrm{m}^{2}\right)$ (Table 1). The most common cause for ESKD was glomerulonephritis (28.9\%), followed by diabetes (26.1\%). There was a high prevalence of diabetes (37.9\%) and cardiovascular disease (34.1\%) at time of commencement of RRT.

Of the 211 patients, 114 (54\%) experienced a peritonitis event, with a median time to peritonitis from commencement of RRT of 9.6 months (IQR 4.4-20.8 months). Females were more likely to have peritonitis compared to males $(64.5 \% \mathrm{v} 48.1 \%, \mathrm{p}=0.02)$. In our cohort, 97 patients (46\%) did not experience peritonitis during the follow up period while 49 patients (23.2\%) experienced 1 episode of peritonitis and 65 patients (30.8\%) experienced 2 or more peritonitis episodes during the follow up period.

\section{CARDIOVASCULAR EVENTS}

A total of $65(30.8 \%)$ patients experienced a cardiovascular event. Total cardiovascular events included death from cardiac causes (32.3\%), myocardial infarction (24.6\%), unstable angina (13.8\%), transient ischaemic attack (9.2\%), cerebrovascular event (9.2\%), death from cerebrovascular event (6.2\%) and heart failure (4.6\%). The median time from commencement of PD to cardiovascular event was 30 months (IQR 15-69.4 months). In our cohort, 35 patients 
(16.1\%) experienced a cardiovascular event after a peritonitis episode, with a median time from peritonitis episode to cardiovascular event of 2.4 months (IQR 1.1-4.6 months). Risk factors for a cardiovascular event included cerebrovascular disease (HR 2.72, 95\% CI 1.365.47, $\mathrm{p}=0.005)$, diabetes $(\mathrm{HR} 2.41,95 \% \mathrm{Cl} 1.47-3.96, \mathrm{p}=0.001)$ and pre-existing coronary artery disease at commencement of RRT (HR 1.67, 95\% Cl 1.01-2.77, p=0.047). Older age increased the risk of cardiovascular events $(\mathrm{HR} 1.03,95 \% \mathrm{Cl} 1.01-1.06, p=0.002)$ which was augmented when age was over 65 (HR 2.02, 95\% Cl 1.21-3.38, p=0.007) (Table 2).

\section{ASSOCIATION OF PERITONITIS WITH CARDIOVASCULAR EVENTS}

Of the 65 patients who experienced a cardiovascular event, 35 (53.8\%) had a preceding episode of peritonitis and $30(46.2 \%)$ did not. There was no evidence that patients with a peritonitis episode had an increased risk of cardiovascular events, when compared to patients with no peritonitis episode $(30.7 \%$ vs $30.9 \%, p=0.97)$. Time dependent covariate analysis showed a similar result (HR 1.37 95\% Cl 0.81-2.32, p=0.24). After accounting for age, cerebrovascular disease, diabetes and existing coronary artery disease, the risk of cardiovascular events from peritonitis remained $1.32(95 \% \mathrm{Cl} 0.78-2.23$, $\mathrm{p}=0.30)$. 


\section{DISCUSSION}

This is the first study to examine peritonitis as a risk factor for all cardiovascular events, in addition to cardiovascular mortality. In our cohort, a large proportion (54\%) of patients experienced at least one episode of peritonitis, with $31 \%$ of patients experiencing a cardiovascular event. Similar to previously established risk factors, we found that a diagnosis of cerebrovascular disease, diabetes and coronary artery disease at the time of commencing RRT, increased the risk of cardiovascular events 2.72, 2.41 and 1.67-fold respectively. Older age also increased the risk of cardiovascular events. However, we did not find an increased risk of cardiovascular events following an episode of peritonitis.

Current theory indicates that peritonitis may predispose patients to cardiovascular events through systemic inflammation and direct inflammatory effects on pre-existing coronary artery atherosclerotic plaques ${ }^{12,19-20}$. CKD patients have accelerated cardiovascular disease from multiple factors including oxidative stress, uraemia, fluid overload and chronic inflammation ${ }^{7-8}$, and therefore are likely to have underlying atherosclerotic lesions. Haemodynamic changes including tachycardia and hypotension in the setting of infection can result in demand ischaemia in the context of pre-existing coronary artery lesions ${ }^{15-16,20}$. Concurrent endothelial dysfunction leading to coronary vasoconstriction and increased direct inflammatory activity within the atherosclerotic lesions, can also lead to plaque rupture ${ }^{10,15-16,20}$. Systemic inflammatory responses with production of cytokines, prothrombotic factors, and vasoactive molecules contribute to thrombus formation ${ }^{10,15-}$ 16,20. Transient peritoneal membrane changes associated with PD peritonitis may also allow diffusion of inflammatory cytokines into the blood leading to systemic inflammation which persists even once the local peritoneal infection settles ${ }^{7}$. 
Infection and cardiovascular risk in the CKD and non-CKD population is now an established association $^{7-8,15-16}$. In the CKD population, a prospective cohort study, demonstrated any infection increased the risk of cardiovascular ischaemia, congestive heart failure and mortality by $1.8,3.2$ and 3.4 times respectively ${ }^{8}$. These findings can be extended to the dialysis population where an infection-related hospitalization increases the risk of cardiovascular events by $18 \% 18$ and an episode of bacteraemia increases the risk of congestive cardiac failure, myocardial infarction and stroke by $1.65,1.78$ and 2.04-fold respectively ${ }^{17}$.

Our findings are contrary to limited evidence in the literature which suggest that PD peritonitis is associated with increased cardiovascular mortality. A recent study examining a Brazilian cohort of PD patients suggested peritonitis was associated with a $22 \%$ increased risk of cardiovascular mortality? However, the study was limited to cardiovascular mortality only, while we attempted to capture all cardiovascular events. This study also had a significantly lower peritonitis frequency (26\%) compared to other studies including; a single centre Dutch Study with a peritonitis frequency of $72 \%^{21}$, an Australian study with a frequency of $56 \%^{19}$, as well as our study (54\%). An ANZDATA study also demonstrated an increased risk of mortality, with 19\% of PD patients dying after an episode of peritonitis within the preceding 30 days, with patients dying of vascular disease (cardiovascular, cerebrovascular or peripheral vascular disease) being 3.4 times more likely to have had peritonitis in the preceding 30 days $^{19}$. Despite this the median time from peritonitis to death was 247 days (IQR 64-552) ${ }^{19}$, suggesting the method of case-crossover design may not have completely examined the long-term impact infection may have on cardiovascular risk.

There are a number of reasons for our unexpected findings. Our study was a retrospective analysis utilising records from a single health district. This resulted in a modest sample size which 
may have underpowered our study for a statistically significant result. There is also the possibility that the effect of peritonitis, , as a risk factor for cardiovascular events is attenuated given emphasis on rapid recognition and early initiation of antibiotics ${ }^{22}$. Previous studies examining the association between infection and cardiovascular events in the CKD population relied on established infection, often requiring hospitalisation or the presence of a positive blood culture ${ }^{8,17-}$ 18. A reduction in the severity of the infection episode by rapid recognition and treatment may mitigate the severe inflammatory response and haemodynamic changes attenuating the risk of cardiovascular events ${ }^{15-16,20}$. However larger studies are required to further examine this association.

The main strength of this study was the use of a national registry (ANZDATA) with standardised data collection, as well as the Centre for Health Research Illawarra Shoalhaven Population's (CHRISP) data-linkage capacity which facilitated capturing event data. We were also the first, to the best of our knowledge, to examine PD peritonitis as a risk factor for all cardiovascular events including cardiovascular mortality. In addition to these strengths, our study also had some limitations which need to be acknowledged. The ANZDATA registry is a voluntary, clinician driven registry with no external examination of data accuracy or requirement for standardized definitions of diagnoses, including peritonitis episodes. Information regarding PD patients may be missing from the database (e.g. peritonitis episodes, comorbidities) and inaccurate information may have been entered (e.g. cause of death, date of peritonitis episode). The ANZDATA database does not provide information on the severity of infection including markers of inflammation e.g. C-reactive protein, or other risk factors for cardiovascular disease including hypertension, dyslipidaemia and glycaemic control, residual urine output, dialysis adequacy or patient compliance; which may result in unidentified associations. Lack of data on the use of cardioprotective medication including ACE inhibitor, angiotensin receptor antagonists and HMG- 
CoA reductase inhibitors was also a limitation. The use of ICD-10 and ACHI coding to identify cardiovascular events and procedures, rather than clinical data, may have also led to underestimation of the event rate. However, these limitations are inherent to all studies relying on registries $(5,18-19)$. As discussed above our study may also be underpowered. However, given the retrospective design and reliance on registry data it would not be possible to recruit more patients. Finally, we did not account for other intercurrent infections (e.g. blood stream infections or pneumonia) which may have influenced the results.

\section{ACKNOWLEDGEMENTS}

The authors acknowledge the CHRISP research partnership established between the Illawarra Shoalhaven Local Health District (ISLHD) and the University of Wollongong, and ISLHD for funding support and as the source of data used in this study. We acknowledge the Australian and New Zealand Dialysis and Transplant registry (ANZDATA) for assisting with the data acquisition, as well as all of the contributors who voluntarily provide information to the registry.

\section{CONCLUSIONS}

Infection is associated with cardiovascular risk, and the risk of infection in CKD remains higher than the general population. Despite this, our study did not find evidence of an association between peritonitis and risk of cardiovascular events. This may be due to the modest size of our study. However, it is possible that rapid recognition and treatment of peritonitis may mitigate this risk. Larger studies are required to further examine this important association. 


\section{REFERENCES}

1. Australian Institute of Health and Welfare. Australia's health 2018. Australia's health series no.

16. Canberra, Australia: AlHW; 2018. Available from https://www.aihw.gov.au/reports/australias$\underline{\text { health/australias-health-2018/contents/table-of-contents }}$

2. Australia and New Zealand Dialysis and Transplant (ANZDATA) Registry. 41st Report, Chapter

1: Incidence of End Stage Kidney Disease. Adelaide, Australia: ANZDATA Registry; 2018. Available from https://www.anzdata.org.au/report/anzdata-41st-annual-report-2018-anzdata/

3. ANZDATA Registry. 41st Report, Chapter 5: Peritoneal dialysis. Adelaide, Australia: ANZDATA registry; 2018. Available from https://www.anzdata.org.au/report/anzdata-41st-annual-report-2018$\underline{\text { anzdatal }}$

4. Chaudhary K, Sangha H, Khanna R. Peritoneal Dialysis First: Rationale. Clin J Am Soc Nephrol 2011; 6: 447-56.

5. Johnson DW, Dent H, Hawley CM, McDonald SP, Rosman JB, Brown FG, et al. Association of dialysis modality and cardiovascular mortality in incident dialysis patients. Clin J Am Soc Nephrol 2009; 4: 1620-28.

6. ANZDATA Registry. 41st Report, Chapter 3: Mortality in End Stage Kidney Disease. Adelaide, Australia: ANZDATA registry, 2018. Available from https://www.anzdata.org.au/report/anzdata41st-annual-report-2018-anzdata/ 
7. Pecoits-filho R, Yabumoto FM, Campos LG, Moraea TP, Figueiredo AE, Olandoski M, et al. Peritonitis as a risk factor for long-term cardiovascular mortality in peritoneal dialysis patients: The case of a friendly fire? Nephrology 2018; 23: 253-58.

8. Cheikh Hassan HI, Tang M, Djurdjev O, Langsford D, Sood MM, Levin A. Infection in advanced chronic kidney disease leads to increased cardiovascular events, end stage kidney disease and mortality. Kidney Int 2016; 90: 897-904.

9. Go AS, Chertow GM, Fan D, McCulloch CE, Hsu CY. Chronic kidney disease and the risks of death, cardiovascular events and hospitalisation. N Engl J Med 2004; 351: 1296-1305.

10. Stenvinkel P, Pecoits-Filho R, Lindholm B. Coronary artery disease in end stage renal disease: No longer a simple plumbing problem. J Am Soc Nephrol 2003; 14:1927-39.

11. Liakopoulos V, Roumeliotis S, Gorny X, Eleftheriadis T, Mertens PR. Oxidative Stress in Patients undergoing peritoneal dialysis: A current review of the literature. Oxidative medicine and cellular longevity, 2017 [cited 28 Oct 2019]; 3494867. Available from http://downloads.hindawi.com/journals/omcl/2017/3494867.pdf

12. Viasus D, Garcia-Vidal C, Cruzado JM, Adamuz J, Verdaguer R, Manresa F, et al. Epidemiology, clinical features and outcomes of pneumonia in patients with chronic kidney disease. Nephol Dial Transplant 2011; 26: 2899-2906. 
13. McDonald HI, Thomas SL, Nitsch D. Chronic kidney disease as a risk factor for acute community-acquired infections in high-income countries: a systematic review. BMJ open 2014 [Cited 28 Oct 2019]; 4:e004100. Available from https://bmjopen.bmj.com/content/4/4/e004100.long

14. Wang HE, Gamboa C, Warnock DG, Muntner P. Chronic kidney disease and risk of death from infection. Am J Nephrol 2011; 34: 330-36.

15. Corrales-Medina VF, Suh KN, Rose G, Chirinos JA, Doucette S, Cameron DW, et al. Cardiac complications in patients with community-acquired pneumonia: A systematic review and metaanalysis of observational studies. PLoS Medicine 2011 [Cited 28 Oct 2019]; 8(6):e1001048. Available from https://journals.plos.org/plosmedicine/article?id=10.1371/journal.pmed.1001048

16. Corrales-Medina VF, Serpa J, Rueda AM, Giordano TP, Bozkurt B, Madjid M, et al. Acute bacterial pneumonia is associated with the occurrence of acute coronary syndromes. Medicine (Baltimore) 2009; 88: 154-59.

17. Ishani A, Collins AJ, Herzog CA, Foley RN. Septicaemia, access and cardiovascular disease in dialysis patients: The URDS Wave 2 study. Kidney Int 2005; 68: 311-18.

18. Dalrymple LS, Mohammed AM, Mu Y, Johansen KL, Chertow GM, Grimes B, et al. Risk of cardiovascular events after infection-related hospitalizations in older patients on dialysis. Clin J Am Soc Nephrol 2011; 6: 1708-13. 
19. Boudville B, Kemp A, Clayton P, Lim W, Badve SV, Hawley CM, et al. Recent peritonitis associates with mortality among patients treated with peritoneal dialysis. Clin J Am Soc Nephrol 2012; 23:1398-1405.

20. Corrales-Medina VF, Madjid M, Musher DM. Role of acute infection in triggering acute coronary syndromes. Lancet 2010; 10:83-92.

21. Van Esch S, Krediet RT, Struijk DG. 32 years' experience of peritoneal dialysis-related peritonitis in a university hospital Perit Dial Int 2014; 34(2):162-70.

22. Li PK, Szeto CC, Piraino B, de Arteaga J, Fan S, Figueiredo AE, et al. ISPD Peritonitis recommendations: 2016 update on prevention and treatment. Perit Dial Int 2016; 36(5):481-508. 


\section{FIGURE LEGENDS}

Figure 1: Study population including reasons for exclusion

$\mathrm{PD}=$ peritoneal dialysis 


\section{TABLES}

Table 1: Baseline characteristics stratified by peritonitis versus no peritonitis group

Peritonitis

\begin{tabular}{|c|c|c|c|c|}
\hline Variable & Overall & Yes & No & $p$ value \\
\hline$N(\%)$ & 211 & $114(54.0)$ & $97(45.8)$ & \\
\hline Age, years (IQR) & 66.30 (54.49- 74.45) & $66.74(56.25-74.07)$ & $65.45(51.48-75.35)$ & 0.7 \\
\hline Age $\geq 65, n(\%)$ & $111(52.6)$ & $62(54.4)$ & $49(50.5)$ & 0.6 \\
\hline Male, $n(\%)$ & $135(64.0)$ & $65(57.0)$ & $70(72.2)$ & 0.02 \\
\hline Caucasian, $n(\%)$ & $199(94.3)$ & 105 (92.1) & $94(96.9)$ & 0.1 \\
\hline $\mathrm{BMI}, \mathrm{kg} / \mathrm{m}^{2}(\mathrm{SD})$ & $28.1(5.1)$ & $28.4(5.4)$ & $27.7(4.9)$ & 0.4 \\
\hline$<25, n(\%)$ & $60(28.6)$ & $31(27.4)$ & $29(29.9)$ & 0.2 \\
\hline $25-29.9, n(\%)$ & $85(40.5)$ & $41(36.3)$ & $44(45.4)$ & \\
\hline$\geq 30, n(\%)$ & $65(31.0)$ & $41(36.3)$ & $24(24.7)$ & \\
\hline \multicolumn{5}{|l|}{ Kidney disease, $n(\%)$} \\
\hline Diabetes & $55(26.1)$ & $26(22.8)$ & $29(29.9)$ & 0.4 \\
\hline HTN & $33(15.6)$ & $20(17.5)$ & $13(13.4)$ & \\
\hline GN & $61(28.9)$ & $35(30.7)$ & $26(26.8)$ & \\
\hline Cystic & $14(6.6)$ & $5(4.4)$ & $9(9.3)$ & \\
\hline Other & $48(22.7)$ & $28(24.6)$ & $20(20.6)$ & \\
\hline Creatinine, $\mu \mathrm{mol} / \mathrm{l}(\mathrm{IQR})$ & 590 (469.0-744.0) & $604.5(475.0-778.5)$ & 575 (449.0-736.5) & 0.6 \\
\hline $\begin{array}{l}\text { Calcium }(n=169), \mathrm{mmol} / \mathrm{l} \\
(\mathrm{SD})\end{array}$ & $2.31(0.43)$ & $2.28(0.19)$ & $2.33(0.58)$ & 0.4 \\
\hline $\begin{array}{l}\text { Phosphate }(n=169) \text {, } \\
\mathrm{mmol} / / \mathrm{SD})\end{array}$ & $1.68(0.50)$ & $1.65(0.47)$ & $1.70(0.53)$ & 0.6 \\
\hline $\begin{array}{l}\text { Haemoglobin }(n=205), g / l \\
(\mathrm{SD})\end{array}$ & $115.6(15.59)$ & $114.3(14.95)$ & $117.08(16.24)$ & 0.2 \\
\hline \multicolumn{5}{|l|}{ Smoking, $n(\%)$} \\
\hline Current & $26(12.3)$ & $13(11.4)$ & $13(13.4)$ & 0.6 \\
\hline Former & $99(46.9)$ & $57(50.0)$ & $42(43.3)$ & \\
\hline Never & $86(40.8)$ & $44(38.6)$ & $42(43.3)$ & \\
\hline $\begin{array}{l}\text { Chronic Lung disease, } n \\
(\%)\end{array}$ & $17(8.1)$ & $8(7.0)$ & $9(9.3)$ & 0.5 \\
\hline $\begin{array}{l}\text { Coronary artery disease, } n \\
(\%)\end{array}$ & $72(34.1)$ & $36(31.6)$ & $36(37.1)$ & 0.4 \\
\hline Peripheral vascular & $40(19.0)$ & $19(16.7)$ & $21(21.6)$ & 0.4 \\
\hline
\end{tabular}


disease, $n(\%)$

Cerebrovascular disease, $n(\%)$
$27(12.8)$

$80(37.9)$

$65(31.1)$
$15(13.2)$

$43(37.7)$

$37(32.7)$
$12(12.4)$ 0.9

Diabetes, $n(\%)$

Cancer $(n=219), n(\%)$

Continuous variables are expressed as mean (SD) or median (IQR). BMI=Body Mass Index.

Table 2: Univariate Cox Regression analysis for hazard ratio of cardiovascular event

\begin{tabular}{|c|c|c|}
\hline Variable & Hazard Ratio (HR) [95\% Cl] & $p$ value \\
\hline Age (years) & $1.03[1.01,1.06]$ & 0.002 \\
\hline Age $\geq 65$ (years) & $2.02[1.21,3.38]$ & 0.007 \\
\hline Male & $0.92[0.56,1.51]$ & 0.73 \\
\hline \multicolumn{3}{|l|}{ BMI (<25kg/m² ref) } \\
\hline$<25$ & Reference & 0.48 \\
\hline $25-29.9$ & 0.74 [0.42. 1.33] & \\
\hline$\geq 30$ & $0.71[0.38,1.31]$ & \\
\hline Creatinine $\mathrm{mmol} / \mathrm{L}$ & $0.99[0.99,1.00]$ & 0.17 \\
\hline Calcium mmol/L & $0.67[0.14,3.09]$ & 0.6 \\
\hline Phosphate $\mathrm{mmol} / \mathrm{L}$ & $0.95[0.52,1.75]$ & 0.88 \\
\hline Haemoglobin $\mathrm{g} / \mathrm{L}$ & $1.00[0.98,1.02]$ & 0.74 \\
\hline Chronic lung disease & $1.26[0.54,2.92]$ & 0.6 \\
\hline Coronary artery disease & $1.67[1.01,2.77]$ & 0.047 \\
\hline Peripheral vascular disease & $1.64[0.92,2.93]$ & 0.096 \\
\hline Cerebrovascular disease & $2.72[1.36,5.47]$ & 0.005 \\
\hline Diabetes Mellitus & $2.41[1.47,3.96]$ & 0.001 \\
\hline Cancer & $0.74[0.43,1.27]$ & 0.27 \\
\hline
\end{tabular}

$95 \% \mathrm{Cl}=95 \%$ confidence interval, $\mathrm{BMI}=$ body mass index. 
262 patients who commenced PD between 2001 and 2005

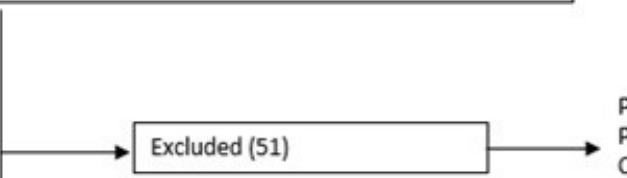

$\mathrm{PD}<3$ months (22)

Previous transplant (1)

Own function recovered (9)

Prior haemodialysis $>6$ months (4)

Commenced PD in another area health service (12)

Never on PD (2)

This article is protected by copyrighteAls rights reserved.

211 patients included for analysis 